\title{
Movement Control Based on Model Predictive Control with Disturbance Suppression using Kalman Filter including Disturbance Estimation
}

\author{
Takashi Ohhira ${ }^{* a)}$ Non-member, Akira Shimada** Senior Member
}

(Manuscript received April 17, 2017, revised April 3, 2018)

\begin{abstract}
This study proposes a movement control system based on model predictive control (MPC), and a Kalman filter (KF) that can consider the influences of noise and disturbance. The KF estimates not only the motion state but also the disturbance of the controlled objects affected by noise. Disturbance is introduced by the stationary disturbance, by the system noise, and by observation noise. An MPC system filtered by the KF is robust and suppresses disturbances using the special design method proposed in this study. The feasibility of the MPC-based control system is confirmed under conditions of strong intermittent disturbances, such as road surface and sensor noises, and a friction force with less time variation. Finally, the proposed method is tested in simulations of a cart traveling in a straight line. The superior simulation results over the existing MPC system validate the proposed control system.
\end{abstract}

Keywords: model predictive control (MPC), Kalman filter (KF), disturbance estimation, disturbance suppression, motion control, multivariable control

\section{Introduction}

Many modern societies are characterized by aging populations with fewer births than previous generations. Therefore, personal robots that can work alongside humans have attracted increasing attention, and various personal robots have been deployed for practical use. Future issues regarding personal robots require control systems to ensure their safety and stability. Constraints are important parameters in the control systems of personal robots.

One suitable control technique is the model predictive control (MPC) technique ${ }^{(1)-(3)}$, which can handle multiinput/multi-output (MIMO) systems and predict future events based on mathematical models of controlled objects. MPC systems can optimize control while imposing upper and lower limits on state quantities and control inputs. However, MPC requires a large amount of numerical optimization at each sampling period and its mathematical models must be highly accurate. Despite these disadvantages, MPC has attracted significant attention in recent years for its potential applications to robotic control. The reason for this is that MPC has been successful in the process control field based on improvements in computational performance. MPC ensures safety and stability by constraining the states of controlled objects. In a previous study on applying an MPC to a personal robot ${ }^{(1)}$, the MPC outperformed conventional control systems by applying constraints to orientation stabilization. This result indicates that controllers considering constraints

\footnotetext{
a) Correspondence to: Takashi Ohhira. E-mail: ma16021@ shibaura-it.ac.jp

* Graduate School of Shibaura Institute of Technology

3-9-14, Shibaura, Minato-ku, Tokyo 108-8548, Japan

${ }^{* *}$ Department of Engineering and Design, Shibaura Institute of Technology

3-9-14, Shibaura, Minato-ku, Tokyo 108-8548, Japan
}

are suitable for advanced personal robot controllers. However, the advantages of MPC are compromised by the influences of noise and disturbances. This problem is known as constraint violation of the MPC ${ }^{(3)}$. Under constraint violation conditions, MPC cannot ensure safe and reliable robot control because it cannot accurately constrain the robot. In standard MPC, disturbances are removed by an integrator. However, because of the slow removal speed of an integrator, disturbances can lead to tracking errors in MPC. Typically, constraint violations are compensated for by relaxing constraints, but this countermeasure significantly reduces benefits of MPC. To suppress or remove disturbances, many researchers have incorporated a disturbance observer (DOB) into $\mathrm{MPC}^{(2)(4)(5)}$. The DOB performs early disturbance estimation and ensures a robust control system by directly removing the estimated disturbance values. However, the DOB cannot always attain the expected performance in the presence of large noise, meaning it is inapplicable in very noisy environments. Previously, we adopted the Kalman filter (KF) in a method for estimating disturbed states ${ }^{(6)}$. Unlike conventional methods, the KF can estimate disturbances in noisy environments and reduce the effect of intermittent noise. Unfortunately, KF-based disturbance estimation is slow, meaning tracking errors remain in the observed values because of the influence of disturbances. To correct this problem, this study exploits the advantages of MPC.

This paper presents an MPC with a KF that can handle the influences of both noise and disturbances. MPC can also handle MIMO systems, which is naturally advantageous for suppressing disturbance. Details regarding this point are presented in Section 3.2. In this study, we design an MPC system that reduces the likelihood of constraint violation in the presence of noise and disturbances. We also design an MPC with disturbance suppression. The proposed system is verified through simulation experiments. 


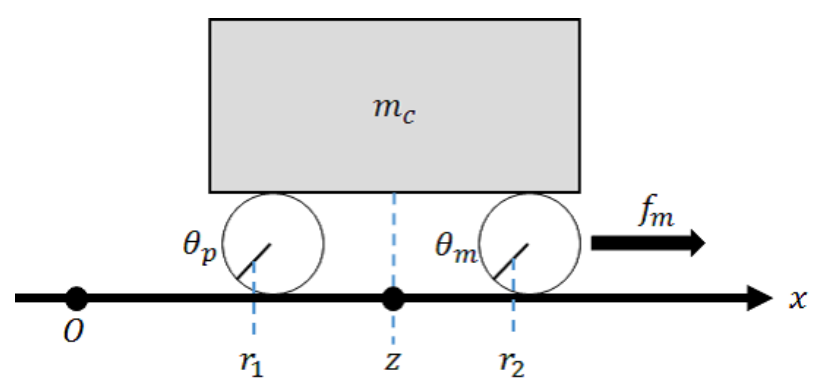

Fig. 1. Diagram for cart modeling

Table 1. Specifications of the cart

\begin{tabular}{c|c}
\hline$m_{c}$ & Mass of cart \\
\hline$z$ & Position of cart \\
\hline$r_{1}$ & Radius of front wheel \\
\hline$r_{2}$ & Radius of rear wheel \\
\hline$\theta_{p}$ & Rotation angle of front wheel \\
\hline$\theta_{m}$ & Rotation angle of rear wheel \\
\hline$C_{c}$ & Viscous friction of cart \\
\hline$C_{m}$ & Viscous friction of motor \\
\hline$\tau_{e x}$ & Force from outside \\
\hline$\tau$ & Motor torque \\
\hline$J$ & Motor moment of inertia \\
\hline$f_{m}$ & Driving force of cart \\
\hline
\end{tabular}

\section{Description and Mathematical Modeling}

This section presents the details of the system to be controlled. MPC prediction and KF estimation are governed by a mathematical model of the controlled system. The controlled system in this study is a cart. The parameters of the mathematical model are defined in Fig. 1 and Table 1.

Equations (1) and (2) define the position of the cart (z).

$$
\begin{aligned}
& z=r_{1} \theta_{p} \\
& z=r_{2} \theta_{m}
\end{aligned}
$$

The driving force is given by

$$
f_{m}=\frac{\tau}{r_{2}}
$$

The motion equations of the motor and cart are given by Eqs. (4) and (5), respectively.

$$
\begin{aligned}
& J \ddot{\theta}_{m}+C_{m} \dot{\theta}_{m}=\tau-\tau_{e x} \\
& m_{c} \ddot{z}+C_{c} \dot{z}=\frac{\tau_{e x}}{r_{2}} \ldots \ldots
\end{aligned}
$$

From Eq. (2), we have

$$
\theta_{m}=\frac{z}{r_{2}}
$$

Substituting Eq. (6) into Eq. (3), we obtain

$$
\frac{J}{r_{2}} \ddot{z}+\frac{C_{m}}{r_{2}} \dot{z}=\tau-\tau_{e x}
$$

Dividing both sides of Eq. (7) by $r_{2}$ gives

$$
\frac{J}{r_{2}^{2}} \ddot{z}+\frac{C_{m}}{r_{2}^{2}} \dot{z}=\frac{\tau}{r_{2}}-\frac{\tau_{e x}}{r_{2}} .
$$

Adding Eqs. (5) and (8), we then obtain

$$
\left(m_{c}+\frac{J}{r_{2}^{2}}\right) \ddot{z}+\left(C_{c}+\frac{C_{m}}{r_{2}^{2}}\right) \dot{z}=\frac{\tau}{r_{2}}=f_{m} .
$$

Defining $m_{c}+\frac{J}{r_{2}^{2}}=M$ and $C_{c}+\frac{C_{m}}{r_{2}^{2}}=C$, we can write

$$
M \ddot{z}+C \dot{z}=f_{m} . \cdots \ldots \ldots \ldots \ldots \ldots \ldots \ldots \ldots \ldots \ldots \ldots \ldots \ldots \ldots
$$

We now derive the state space model of the cart. The action of the cart is defined by five parameters; mass $(=M)$, viscous friction $(=C)$, cart position $(=z)$, cart velocity $(=v)$ and the state variable $\left(x=\left[\begin{array}{ll}z & v\end{array}\right]^{T}\right)$.

From Eq. (10), we have

$$
\frac{d v}{d t}=\dot{v}=-\frac{C}{M} v+\frac{1}{M} f_{m}
$$

The state and output equations are respectively given by

$$
\dot{x}=\left[\begin{array}{l}
\dot{z} \\
\dot{v}
\end{array}\right]=\left[\begin{array}{rr}
0 & 1 \\
0 & -\frac{C}{M}
\end{array}\right]\left[\begin{array}{l}
z \\
v
\end{array}\right]+\left[\begin{array}{c}
0 \\
\frac{1}{M}
\end{array}\right] f_{m} \ldots
$$

and

$$
y=z=\left[\begin{array}{ll}
1 & 0
\end{array}\right]\left[\begin{array}{l}
z \\
v
\end{array}\right]
$$

\section{Proposed Control System}

This section presents the details of the proposed control system. The configuration of the proposed system is illustrated in Fig. 2. The general MPC system is comprised of an optimizer that calculates the optimal amount of control input and a predictor that calculates the predicted trajectory of the state and controller input. In addition to the general MPC system, the proposed system includes an additional "Kalman filter with disturbance estimation", which estimates the state of the cart. The MPC and KF designs are based on the state equations of an enlargement system $\left(\bar{X}=\left[\begin{array}{lll}z & v & \hat{d}\end{array}\right]^{T}\right)$. The enlargement system evolves the state variables, including the disturbance estimation values $(\hat{d})$. The observation matrices for the MPC and KF are independently designed. When only the position is observable, the KF observation matrix is given by $\left(C=\left[\begin{array}{lll}1 & 0 & 0\end{array}\right]\right)$. Based on the KF estimate, the observation matrix for MPC is $\left(C=I^{3 \times 3}\right)$. The MPC handles all observations of the state variables in the controlled system and assigns a set-point value to the disturbance. Through this operation, the proposed method controls disturbances in a pseudo-type manner and suppresses their influence through the MPC. In the proposed method, all disturbances other than stationary disturbance are regarded as system noise. Such disturbances include system and observation noises, such as the road surface and friction noise. The KF with disturbance estimation attenuates the system and observation noises.

\subsection{Kalman Filter with Disturbance Estimation}

We define the stationary disturbance as $d(k)$. The other variables are the state variable $x(k)=\left[\begin{array}{ll}z & v\end{array}\right]^{T}$, control input $u(k)$, control output $y(k)$, and coefficient matrices of the state space model $\left(A_{d}, B_{d}\right.$, and $\left.C_{d}\right)$. The estimated sampling time is $\delta_{1}$. The state space model of the controlled system is then given by

$$
\begin{aligned}
& x(k+1)=\boldsymbol{A}_{d} x(k)+\boldsymbol{B}_{d} u(k)-\boldsymbol{B}_{d} d(k), \ldots \ldots \ldots \ldots \ldots \ldots \ldots \ldots \ldots \ldots \ldots \ldots \ldots \ldots \ldots \ldots \ldots \\
& y(k)=\boldsymbol{C}_{d} x(k) . \cdots \ldots \ldots \ldots \ldots \ldots
\end{aligned}
$$




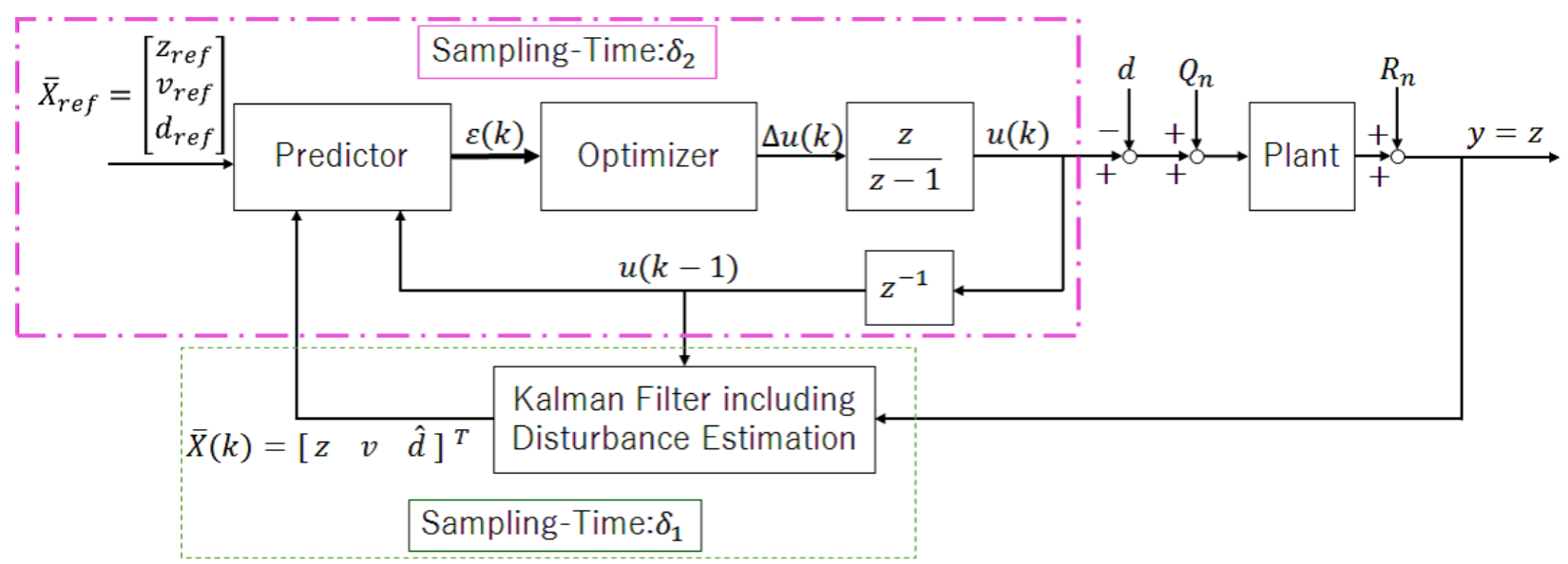

Fig. 2. Block diagram of MPC with a Kalman filter for disturbance estimation

The state space model of the enlargement system $(\bar{X}=$ $\left.\left[\begin{array}{ll}x & \hat{d}\end{array}\right]^{T}\right)$ is written as

$$
\begin{aligned}
& {\left[\begin{array}{l}
x(k+1) \\
d(k+1)
\end{array}\right]=\left[\begin{array}{cc}
\boldsymbol{A}_{d} & -\boldsymbol{B}_{d} \\
0 & \boldsymbol{I}
\end{array}\right]\left[\begin{array}{c}
x(k) \\
d(k)
\end{array}\right]+\left[\begin{array}{c}
\boldsymbol{B}_{d} \\
0
\end{array}\right] u(k), \ldots \ldots} \\
& y(k)=\left[\begin{array}{ll}
\boldsymbol{C}_{d} & 0
\end{array}\right]\left[\begin{array}{l}
x(k) \\
d(k)
\end{array}\right] \ldots \ldots \ldots \ldots \ldots \ldots \ldots \ldots \ldots
\end{aligned}
$$

This enlargement system can estimate both an input and output disturbance. Equations (16) and (17) are utilized in the KF design because the KF considers the influence of disturbances. When system and observation noises following normal white noise disturbances are added to the enlargement system, the state space model becomes

$$
\begin{aligned}
& \bar{X}(k+1)=\overline{A_{d}} \bar{X}(k)+\bar{B}_{d} u(k)+\bar{B}_{d} v(k), \ldots \ldots \ldots \\
& y(k)=\bar{C}_{d} \bar{X}(k)+w(k), \ldots \ldots \ldots \ldots \ldots \ldots \ldots \ldots \ldots
\end{aligned}
$$

$\overline{A_{d}}=\left[\begin{array}{cc}\boldsymbol{A}_{d} & -\boldsymbol{B}_{d} \\ 0 & \boldsymbol{I}\end{array}\right], \bar{B}_{d}=\left[\begin{array}{c}\boldsymbol{B}_{d} \\ 0\end{array}\right]$ and $\bar{C}_{d}=\left[\begin{array}{ll}\boldsymbol{C}_{d} & 0\end{array}\right]$.

The design method of the KF is detailed in ${ }^{(7)}$.

State estimation by the KF proceeds based on the following time-update equations: (Eqs. (20)-(24)).

$$
\begin{aligned}
& \hat{X}^{-}(k)=\overline{A_{d}} \hat{X}(k-1)+\bar{B}_{d} u(k-1) \cdots \ldots \ldots \ldots \ldots \\
& P^{-}(k)=\overline{A_{d}} P(k-1) \bar{A}_{d}^{T}+\bar{B}_{d} \sigma_{v}^{2} \bar{B}_{d}^{T} \ldots \ldots \ldots \ldots \\
& G(k)=P^{-}(k) \bar{C}_{d}\left(\bar{C}_{d} P^{-}(k) \bar{C}_{d}^{T}+\sigma_{w}^{2}\right)^{-1} \ldots \ldots \ldots \ldots \\
& \hat{X}(k)=\hat{X}^{-}(k)+G(k)\left(y(k)-\bar{C}_{d} \hat{X}^{-}(k)\right) \ldots \ldots \ldots \ldots \\
& P(k)=\left(I-G(k) \bar{C}_{d}\right) P^{-}(k) \ldots \ldots \ldots \ldots \ldots \ldots
\end{aligned}
$$

where $\hat{X}^{-}(k)$ and $P^{-}(k)$ are the a priori state estimation and a priori covariance matrix respectively, and $\hat{X}(k)$ and $P(k)$ are the corresponding a posteriori state estimation and covariance matrix, respectively.

$G(k)$ is the Kalman gain, and $\sigma_{v}^{2}$ and $\sigma_{w}^{2}$ are the variances of the system and observation noises, respectively.

Based on Eqs. (20)-(24)), the KF with disturbance estimation can be designed using the variables of the enlargement system defined in Eqs. (18) and (19).

3.2 Model Predictive Control (MPC) with Disturbance Suppression In this subsection, we describe only the MPC components that are required by the proposed method. The prediction model is a linear state-space model in discrete time. Here, we only present an overview of the proposed method. Details of our design methodology can be found in ${ }^{(2)(3)(8)(9)}$.

Many studies on MPC considering disturbances have been conducted in recent years ${ }^{(10)-(13)}$. As a prerequisite for the proposed method, the disturbance estimates do not directly cancel the disturbances (unlike conventional DOB). By utilizing the enlargement system, we designed a constrained nonlinear optimization problem for the MPC. The designed system suppresses the effects of stationary disturbances. Through this operation, the proposed method can control disturbances in a pseudo-type manner by considering position and velocity. Because the disturbances cannot be controlled or predicted directly, the position, velocity, and disturbance are estimated by the KF, and multiple states (including the disturbances) are optimized by the MPC which derives the control inputs. Therefore, we assign set values by adding the disturbance terms to the objective function. These terms minimize the tracking error of the set and estimated disturbance values. We also design a weight matrix that weights disturbances more lightly than other states. Therefore, when deriving the control input, the tracking error of the disturbance is considered. Through this operation, the MPC of the proposed system suppresses or removes disturbances. The MPC of the proposed method is also applicable to oscillating and vibrating systems in which disturbances are directly canceled.

We now determine the optimized trajectory for the amount of change $\Delta u$ in the control input. To this end, we select the $\Delta u$ for which the "Predicted Trajectory $(X(k), U(k))$ " has the smallest "Tracking Error $(\varepsilon(k))$ " from the "Set-point Trajectory $(T(k))$ " from the present time $k$ to $H_{p}$ steps in the future. The proposed method reduces the computational load of MPC by adopting a multirate sampling control strategy. It also controls all states (including disturbances) and observes all states by utilizing the KF. The state space model for prediction is defined by a new sampling time $\left(\delta_{2}\right)$. The output matrix of the MPC prediction is defined as $C_{d}=I^{3 \times 3}$. However, because the enlargement system is not controllable, the MPC performs feedback control based on the estimated values. In practice, the MPC derives a control input that suppresses the influence of unpredictable and uncontrollable disturbances when controlling the target object. We also define an internal prediction model that utilizes Eqs. (16) and (17), which are defined in subsection 3.1, and is discretized in time. 
The predicted state trajectory from $k$ to the destination $H_{p}$ is given by

$$
Z_{(k)}=\left[\begin{array}{c}
\hat{x}(k+1 \mid k) \\
\vdots \\
\hat{x}\left(k+H_{p} \mid k\right)
\end{array}\right]=\Psi x_{(k)}+\Gamma u_{(k-1)}+\Theta \Delta U_{(k)}
$$

The amount of change in the control input can be determined by solving the following optimization problem:

$$
\begin{aligned}
& \underset{\Delta U}{\operatorname{minimize}} V_{(k)}=-\Delta U^{T}(k) G+\Delta U^{T}(k) H \Delta U(k) \\
& \text { subject to } \Omega \Delta U \leq \omega \ldots \ldots \ldots \ldots \ldots \ldots \ldots \ldots \ldots \ldots
\end{aligned}
$$

Where,

$$
\begin{aligned}
& \Delta U(k)=\left[\begin{array}{c}
\Delta \hat{u}(k \mid k) \\
\vdots \\
\Delta \hat{u}\left(k+H_{u}-1 \mid k\right)
\end{array}\right], \\
& G=2 \cdot \Theta^{T} \cdot Q \cdot \varepsilon(k), \\
& H=\Theta^{T} \cdot Q \cdot \Theta+R,
\end{aligned}
$$$$
\Psi=\left[\begin{array}{c}
A \\
\vdots \\
A^{H_{u}} \\
\vdots \\
A^{H_{p}}
\end{array}\right], \Gamma=\left[\begin{array}{c}
B \\
\vdots \\
\sum_{i=0}^{H_{u}} A^{i} B \\
\vdots \\
\sum_{i=0}^{H_{p}-1} A^{i} B
\end{array}\right],
$$

and

$$
\Theta=\left[\begin{array}{ccc}
B & \ldots & 0 \\
A B+B & \ldots & 0 \\
\vdots & \ddots & \vdots \\
\sum_{i=0}^{H_{u}-1} A^{i} B & \ldots & B \\
\sum_{i=0}^{H_{u}} A^{i} B & \ldots & A B+B \\
\vdots & \vdots & \vdots \\
\sum_{p}-1 & & H_{p}-H_{u} \\
\sum_{i=0}^{i} B & \ldots & \sum_{i=0}^{i} A^{i} B
\end{array}\right] .
$$

The variable definitions are based on those in ${ }^{(3)}$ : $\Omega \in \mathbb{R}^{60 \times 5}$ is the constraint matrix.

$\omega \in \mathbb{R}^{60 \times 1}$ is a vector that determines constraint activation.

The diagonal matrix $Q \in \mathbb{R}^{60 \times 60}$ is the weighting matrix for the tracking error.

The diagonal matrix $R \in \mathbb{R}^{5 \times 5}$ is the weighting matrix for the controller input.

$\Omega$ is a matrix of constraints ${ }^{(8)}$. To obtain the matrix $\Omega$, we express the following linear inequalities in matrix form:

$$
\begin{aligned}
& \tau_{\min } \leq u(k+i) \leq \tau_{\max } \quad i=0, \ldots, H_{u}, \\
& x_{\text {min }} \leq x(k+i) \leq x_{\max } \quad i=0, \ldots, H_{p}, \\
& \Delta u_{\text {min }} \leq \Delta u(k+i) \leq \Delta u_{\max } \quad i=0, \ldots, H_{u} .
\end{aligned}
$$

The state and control input are predicted by the mathematical model of the controlled system, which was derived in
Eqs. (16) and (17). Predictions are made within a predefined interval called the prediction horizon $\left(H_{p}\right)$. Control horizon $\left(H_{u}\right)$ determines the allowable amount of input change.

Minimizing the objective function $\left(V_{(k)}\right)$ minimizes the optimal parameter vector $\Delta u(k)$. The matrices $\Psi, \Gamma$ and $\Theta$ are derived from the mathematical prediction model. The predicted trajectories $Z_{(k)}$ and $\Delta U_{(k)}$ represent the input and output changes from $k$ to $H_{p}$, respectively. These trajectories are utilized to solve the objective function. The constraints related to the "state", "control input", and "amount of control input change" are expressed by the linear inequality $\Omega \Delta U_{(k)} \leq \omega$. Therefore, the problem becomes a constrained nonlinear optimization problem. Assuming that a solution exists, this optimization problem imposes control within the specified constraints. The proposed MPC system differs from the conventional MPC system mainly based on the adoption of the enlargement system as an internal model. Therefore, the predicted trajectories of the disturbances are included in the tracking error. Additionally, because the weight values of the estimated disturbance values are included in the weight matrix related to the tracking error, the estimated disturbance values are included in the optimization of the objective function. Therefore, unlike the conventional method, the proposed method considers the influence of disturbances on the control input, which is derived by solving the objective function.

If there is a constraint violation, the optimization problem cannot be solved (i.e., the output is "no solution"). This problem must be resolved by introducing a countermeasure. Among several available methods, this paper implements a countermeasure based on Sequential Quadratic Programming (SQP), which avoids "no solution" results for the optimization problem. The SQP utilized to solve the optimization problem in MPC systems is detailed in ${ }^{(3)}$. Additionally, various mathematical books present the use of SQP as a solver for nonlinear programming problems, including quadratic programming problems ${ }^{(14)(15)}$.

The proposed method is constructed from controller codes implemented in MATLAB2017a, which is a software application created by MathWorks Inc. In this study, we designed the optimizer by utilizing a MATLAB nonlinear programming solver called fmincon ${ }^{(16)}$. The MATLAB solver fmincon can handle the SQP method as a solver option.

Here, we describe only the SQP components that are required as a countermeasure to constraint violation based on ${ }^{(16)}$.

SQP can consider imposed constraints. The first step in this process is to convert inequality constraints into equality constraints by utilizing slack variables. Therefore, the constraints handled by SQP are relaxed constraints from the control problem. The relaxed constraints in this paper are constraints that were converted into equality constraints for the optimization problem of MPC. It is important that these relaxed constraints behave very similarly to the non-relaxed constraints. However, if an optimization problem cannot be solved because of constraint violations, SQP derives a solution from the relaxed equality constraints by utilizing slack variables. Additionally, the relaxed constraints can be derived by utilizing local linear approximations of the original constraints ${ }^{(3)}$. The SQP can facilitate this series of actions. 
Therefore, the proposed method can derive an optimal solution by avoiding the unsolved optimization problem by adopting SQP.

It should be noted that if there are violated constraints when solving the optimization problem with inequality constraints, the SQP method utilizes three ancillary processes ${ }^{(16)}$.

(1) The objective and constraint functions are combined into a single merit function. The merit function indicates how far the current iteration solution is from the optimal solution. The merit function is then minimized according to the relaxed constraints. There is a possibility that a feasible solution is obtained by this process.

(2) Next, the SQP attempts to derive a feasible solution by utilizing quadratic approximation of the constraints. There is a possibility that a feasible solution is obtained.

(3) In the worst case, the SQP algorithm ignores the constraints and descends by small steps in the steepest descent direction within the current iteration. In this case, the SQP algorithm considers the constraints again in the next iteration.

These processes are detailed in ${ }^{(16)}$.

Through this approach, the proposed method can flexibly handle constraint violations. The MPC controller input can always consider real constraints in conjunction with the relaxed constraints derived by SQP. Additionally, constraint violations caused by disturbances and noise are greatly reduced by the KF and its accompanying disturbance estimations. Therefore, the probability of deriving infeasible or unsatisfactory control inputs is reduced in the proposed control system.

Additionally, the objective function derives $\Delta u . \Delta u$ is then utilized by the accumulator (or integrator in a continuous system) to derive the MPC control input. Therefore, $u$ is not directly obtained from the optimization problem. However, to solve the optimization problem, the predicted trajectory of the control input obtained from $u(k-1)$ is included. This predicted trajectory is utilized to optimize the amount of control input change. When the objective function is solved, the control input is taken into account. Therefore, the MPC is a structure that can impose constraints directly on the control inputs. For this reason, optimization including disturbance influences on the amount of control input change can indirectly achieve disturbance suppression of the real control inputs in the proposed MPC system. However, there are cases where there are constraints that must be absolutely satisfied in real environments. In such cases, the constraints on the objective function must be set tighter than the original constraints.

3.3 Multirate Sampling Control (MSC) To reduce sampling time, the proposed method requires state estimation of the controlled system. However, this task places a high computational load on the MPC. Therefore, if the sampling time is too short, the computational complexity exceeds the limits of the processor and the method becomes infeasible. To balance the prediction time and computational load of the MPC with the estimation accuracy for the controlled system, the proposed method utilizes MSC ${ }^{(17)}$. In this approach, the sampling time becomes a tuning parameter for the MPC. We set independent sampling times for the MPC and KF.

For this purpose, we specify two sampling time parameters, namely $\delta_{1}$ for the KF with disturbance estimation and $\delta_{2}$ for the MPC. When $\delta_{2}$ is large, $\delta_{1}$ and $\delta_{2}$ are related by Eq. (27). Both variables must satisfy Eq. (27) as follows:

$$
\delta_{2}=n \cdot \delta_{1}[s]
$$

where $n$ is a positive integer. In this study, we set $n=10$ based on the calculation requirements.

The control input of the MPC is updated with a period of $n \cdot \delta_{1}$ and is constant from $k n \cdot \delta_{1}$ to $(k+1) n \cdot \delta_{1}$. By introducing the MSC, the horizon $\left(H_{p}\right)$ of the predictive time $\left(\delta_{2} \cdot H_{p}[s]\right)$ can be shortened to $(1 / n)$ and the computational load is reduced to $(1 / n)$. Unfortunately, a large sampling time reduces the prediction accuracy of the MPC. To reduce the possibility of accuracy degradation, we adopt a KF with higher estimation accuracy compared to the conventional method in noisy environments.

\section{Simulation Verification}

This section verifies the proposed method through a simulation study of movement control.

The experimental control is based on Eqs. (12) and (13) and was implemented for a cart. The set values were position $1 \mathrm{~m}$, velocity $0 \mathrm{~m} / \mathrm{s}$, disturbance $0 \mathrm{~N} \cdot \mathrm{m}$. The position constraint of the controlled system is $1 \mathrm{~m}$. Under this constraint, the control system can effectively suppress overshoot. The simulation conditions for the cart are listed in Table 2. The cart was subjected to the following disturbances:

1) Disturbance from an uneven road surface such as asphalt,

2) Small sensor noise,

3) An external force after reaching the target position.

These disturbances are often encountered by mobile robots. The intermittent disturbance from the road surface is treated as system noise. In term of verification noise, the sensor noise is much smaller than the road surface noise.

4.1 Tuning Parameters The tuning parameters for MPC and the KF are listed in Tables 3 and 4, respectively. Because disturbances, including noise, are one source of estimation error, the KF design did not accurately estimate noise without disturbance in the simulation. To resolve this problem, we performed the following operations:

Set the covariance of the system noise to 18 times the real system noise,

Set the covariance of the observation noise to 0.09 times the real observation noise,

Set a sufficiently small initial value for the covariance matrix.

The first operation improves the estimation error and response to disturbances, but increases the noise sensitivity of the estimated value. This problem is ameliorated by the second and third operations in the proposed method.

The matrices $Q_{m}$ and $R_{m}$ represent the weighting matrices in the model predictive controller. The entries $Q_{m}$ and $R_{m}$ in $Q_{m p c}$ and $R_{m p c}$, respectively, are appropriately changed

Table 2. Specifications of the verification conditions

\begin{tabular}{c|cc}
\hline Distribution of system noise & \multicolumn{2}{|c}{$Q_{n}=1 e-3$} \\
\hline Distribution of observation noise & \multicolumn{2}{|c}{$R_{n}=2 e-4$} \\
\hline Stationary disturbance & $d=0.5 \quad$ (in $10[s])$ \\
\hline \multicolumn{2}{c}{ where, $e X=10^{X}$}
\end{tabular}


Table 3. Specifications of Kalman filter

\begin{tabular}{c|c}
\hline $\begin{array}{c}\text { Covariance } \\
\text { of system noise }\end{array}$ & $Q_{k l}=Q_{n} \times 18$ \\
\hline $\begin{array}{c}\text { Covariance } \\
\text { of observation noise }\end{array}$ & $R_{k l}=R_{n} \times 0.09$ \\
\hline $\begin{array}{c}\text { Initial value } \\
\text { of covariance matrix }\end{array}$ & $P_{0}=\operatorname{diag}(1 e-61 e-61 e-2)$ \\
\hline $\begin{array}{c}\text { Sampling time } \\
\text { of estimation }\end{array}$ & $\delta_{1}[s]=0.01$ \\
\hline
\end{tabular}

Table 4. Specifications of MPC

\begin{tabular}{c|c}
\hline $\begin{array}{c}\text { Weight matrix } \\
\text { for tracking error }\end{array}$ & $Q_{m}=\operatorname{diag}(2 e 31 e 21 e-2)$ \\
\hline $\begin{array}{c}\text { End of weight matrix } \\
\text { for tracking error }\end{array}$ & $Q_{m-\text { last }}=3 \times Q_{m}$ \\
\hline $\begin{array}{c}\text { Weight matrix } \\
\text { for amount of control input change }\end{array}$ & $R_{m}=1 e-1$ \\
\hline Each horizon & {$\left[H_{p} H_{u}\right]=[205]$} \\
\hline $\begin{array}{c}\text { Sampling time } \\
\text { of control }\end{array}$ & $\delta_{2}[s]=0.1$ \\
\hline
\end{tabular}

based on the prediction horizon. The final weighting matrix [ $\left.Q_{m \text {-last }}\right]$ of $Q_{m p c}$ was set to triple the normal weight. This setting suppresses the control input.

Specifically, we have

$$
\begin{aligned}
& Q_{m p c}=\left[\begin{array}{ccc}
Q_{m} & & 0 \\
& \ddots & \\
0 & & Q_{m \text {-last }}
\end{array}\right] \in \mathbb{R}^{60 \times 60} \\
& \text { and, } R_{m p c}=\left[\begin{array}{ccc}
R_{m} & & 0 \\
& \ddots & \\
0 & & R_{m}
\end{array}\right] \in \mathbb{R}^{5 \times 5} .
\end{aligned}
$$

where the tuning parameters $\left(Q_{f}, R_{f}, Q_{m p c}, R_{m p c}\right.$ and $H_{p}$, $H_{u}$ ) were determined by trial and error. The best parameter set will suppress the control input, improve the tracking performance, and reduce the computational load.

4.2 Verification Results The simulation results are presented in Figs. 3-5. The observation values, estimated values, and initial cart position are presented in Fig. 3. The position and velocity estimates by the KF are presented in Fig. 4 and the disturbance estimates are presented in Fig. 5. The disturbance estimates of the KF and DOB are compared in Fig. 6. Figure 7 clarifies the constraints imposed by the proposed method. Similar verification results utilizing the conventional MPC method are presented in Fig. 8. All the results confirm proper movement control by the proposed method in the presence of noise and disturbances. The results also confirm that proper constraints were imposed by the proposed method. As shown in Fig.3, the estimation error is small in a noisy environment with disturbances. The disturbances affected the results in the vicinity of $10 \mathrm{~s}$. Thereafter, the disturbances were suppressed by the proposed method. Furthermore, no large deviations appear in the tracking control. Figures 4 and 5 confirm accurate state estimation (including disturbances) by the proposed method in a noisy environment. We can conclude that the proposed method can accurately control the movement of the cart under the influence of disturbances. This success can be attributed to the large $Q_{m p c}$ in the MPC design and the larger system noise $Q_{k l}$ in the KF design compared to the actual noise $Q_{n}$.

The proposed method also successfully limited the position

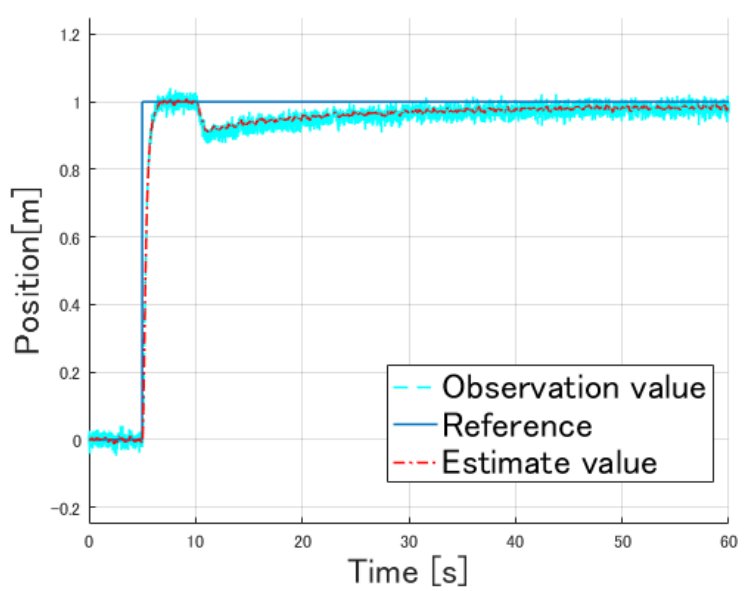

Fig. 3. Simulation results of cart position

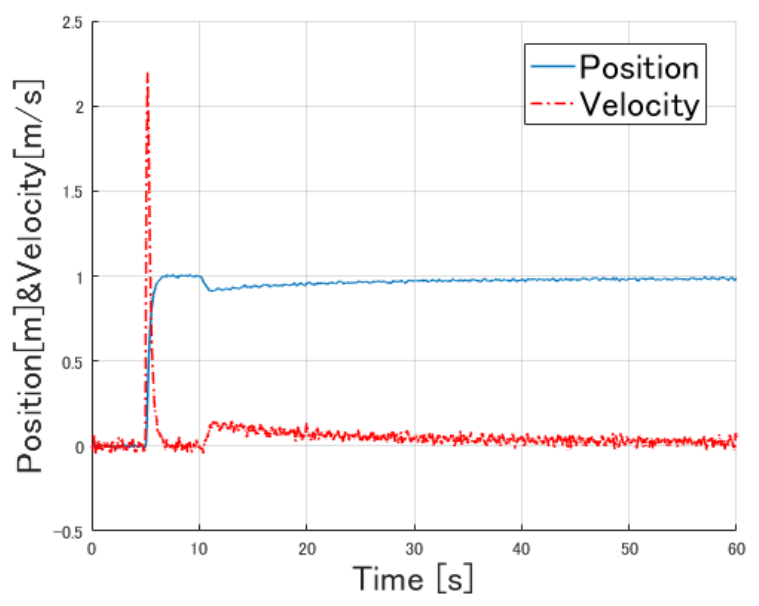

Fig. 4. Simulated position and velocity estimates

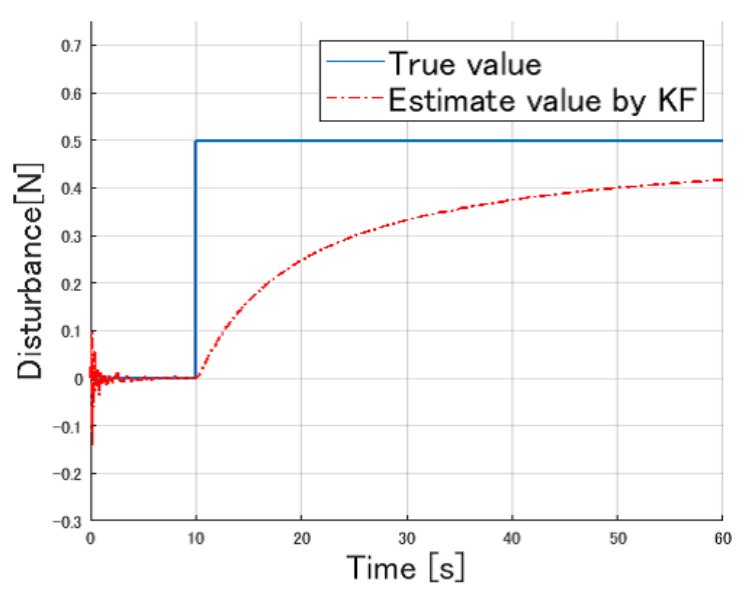

Fig. 5. Simulated disturbance estimation

of the cart (see Figs. 3 and 7). To investigate the effects of constraints on the performance of the proposed method, we constrained the target position by half of the original position constraint. The results of the two trials are compared in Fig. 7. This results indicate proper constraint application by the proposed method. In the conventional MPC system, disturbances and noise led to deviations and oscillations (Fig. 8), and the failure of tracking control. In contrast, the proposed method stably handled the effects of disturbances and noise, demonstrated its superior tracking control. The disturbance 


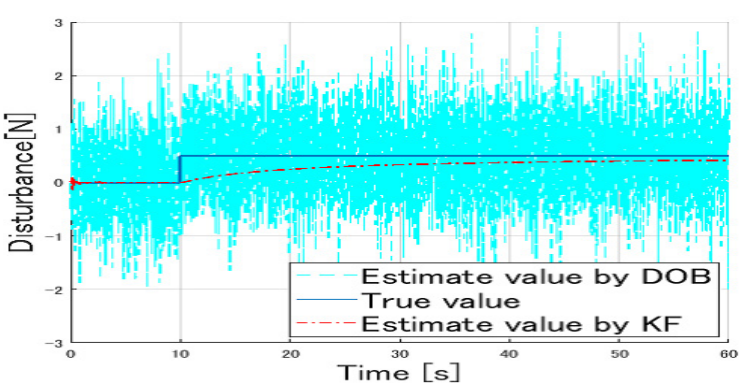

Fig. 6. Comparison of simulated disturbance estimations using $\mathrm{KF}$ and $\mathrm{DOB}$

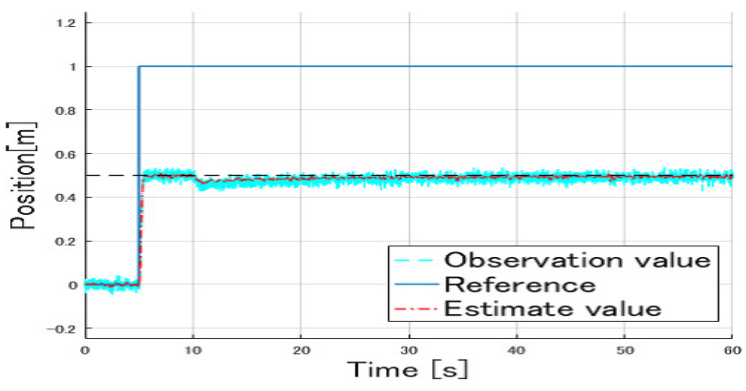

Fig. 7. Simulation results of validation for constraint effectiveness

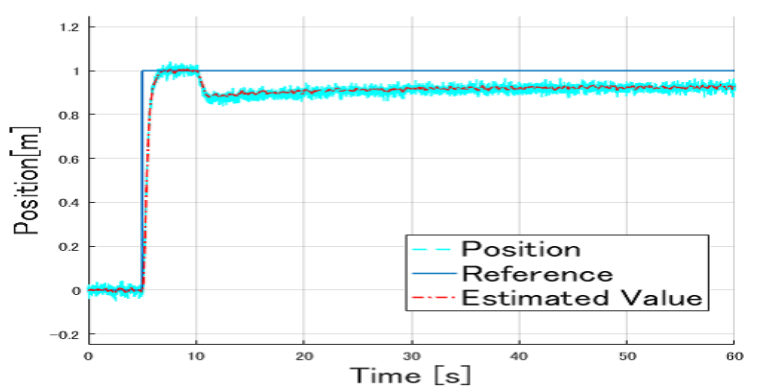

Fig. 8. Simulation results of cart position using conventional MPC

estimations of the KF and DOB are compared in Fig. 6. The initial disturbance estimate and sampling time were the same between both methods. We set the disturbance estimation speed of the proposed KF to be five times faster than that of the existing KF-based disturbance estimation method based on a novel tuning method (described in section 4.1). We assume that the effects of this tuning method are desirable. Although disturbance estimation is slower in the KF than in the DOB, the DOB estimates are strongly influenced by noise. In particular, DOB estimates are prone to vibrations and oscillations. Because the DOB is inapplicable under these conditions, the KF with disturbance estimation is more useful than the DOB in practice. However, the conventional KF with disturbance estimation generated increasing deviations based on its estimation speed. Furthermore, the disturbances resulted in a large estimation error. To solve this problem, the proposed method adopts an enlargement system as the internal MPC model. The MPC of the proposed method can accurately remove disturbances when approximately $80 \%$ of the stationary disturbances can be estimated. The performance of the proposed MPC system is superior to that of conventional MPC systems. Furthermore, the tracking performance of the proposed method is stable after the disturbances are removed.

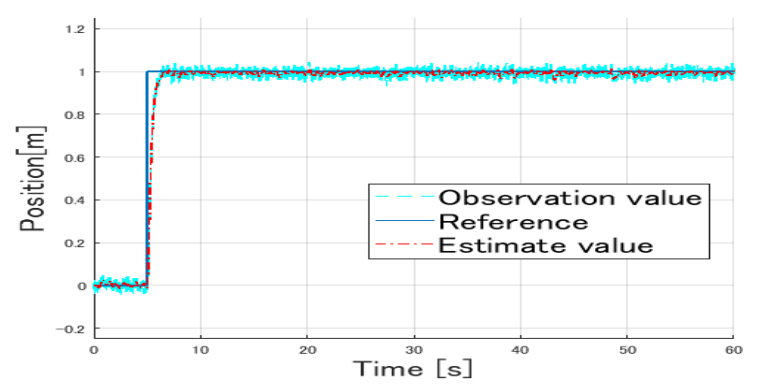

Fig. 9. Simulation results of cart position (disturbance: high frequency sine wave)

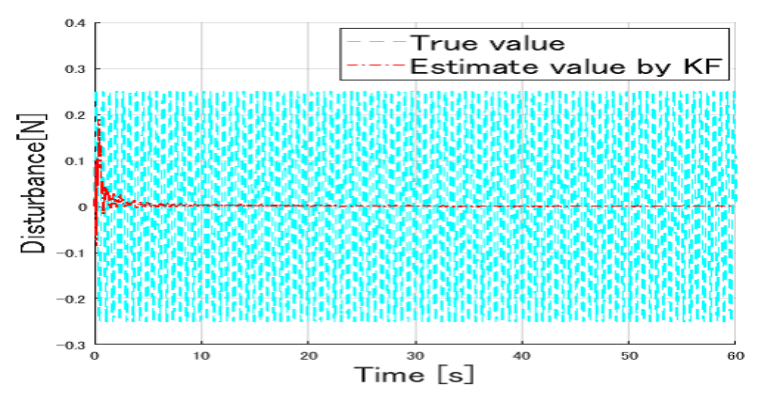

Fig. 10. Simulated disturbance estimation (disturbance: high frequency sine wave)

This fact confirms the superior control performance of the proposed method compared to the conventional MPC system.

However, the performance of the proposed method after disturbance removal depends on the disturbance estimation speed of the KF. From Fig. 5, one can see that the estimated disturbance does not converge to the actual disturbance. Again, the KF with disturbance estimation utilizing the enlargement system has a problem with slow disturbance estimation speed. This estimation speed problem cannot be solved by simply changing some parameters in this study. However, if the disturbance estimation equation of the enlargement system is improved, such as by implementing an equation including the amount of change, the estimated speed may be faster. Additionally, if the disturbance estimation performance of the KF can be improved, the control performance of the proposed method will also be improved.

4.3 Comparative Verification of Disturbances As shown in section 3.1, the enlargement system in this study is a design assuming stationary disturbances (step type disturbances). Therefore, the proposed method is aimed at removing stationary disturbances under the influence of noise. For this reason, the disturbances in the enlargement system are defined as

$$
d(k+1)=d(k)
$$

Therefore, the proposed system cannot guarantee optimal control performances in the presence of disturbances, whose levels the amount change intermittently.

In this section, we verify the performance of the KF, which does not consider the time change of disturbances, in the presence of disturbances. We present comparative verifications of the following disturbances other than step disturbances: high-frequency sine wave $(d=0.25 \sin (10 t))$, lowfrequency sine wave $(d=0.25 \sin (0.2 t))$, pulse wave $(d=$ 0.5 or 0 switching to every $5[\mathrm{~s}])$, and impulse disturbances 


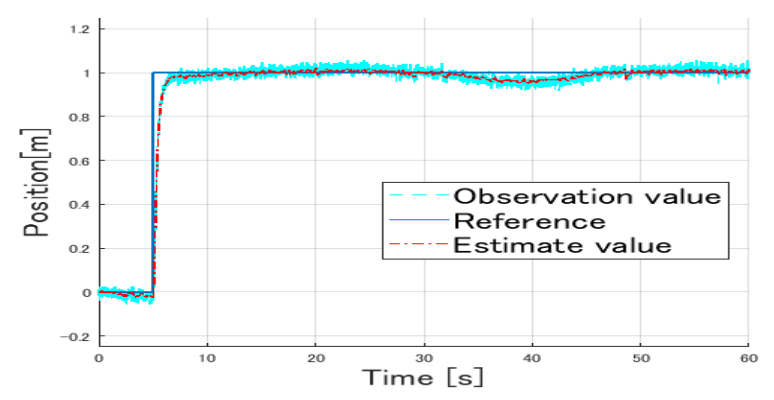

Fig. 11. Simulation results of cart position (disturbance: sine wave)

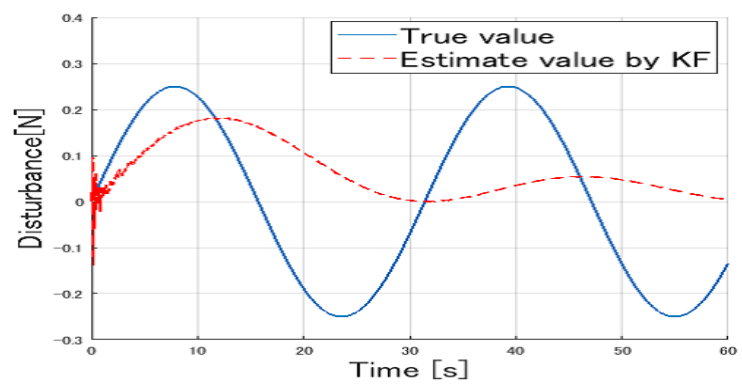

Fig. 12. Simulated disturbance estimation (disturbance: sine wave)

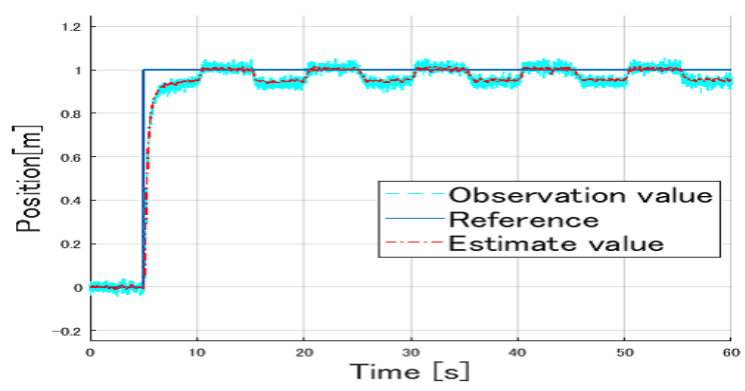

Fig. 13. Simulation results of cart position (disturbance: pulse wave)

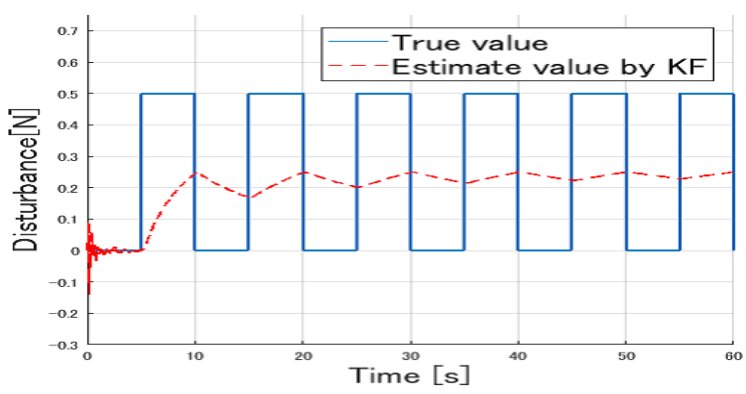

Fig. 14. Simulated disturbance estimation (disturbance: pulse wave)

$(d=100$ in $10[\mathrm{~s}])$.

The comparative results are presented in Figs.9-18. From these results, if a time-varying disturbance is a high frequency in nature, it can be removed as system noise by the KF with disturbance estimation, as shown in Figs. 9 and 10. However, if the time-varying disturbances is low frequency nature, as show in Figs. 11 and 12, it cannot be removed by the proposed system. The reason for this is that low frequency disturbances are treated by the KF as stationary disturbances. Furthermore, in case of disturbances treated by

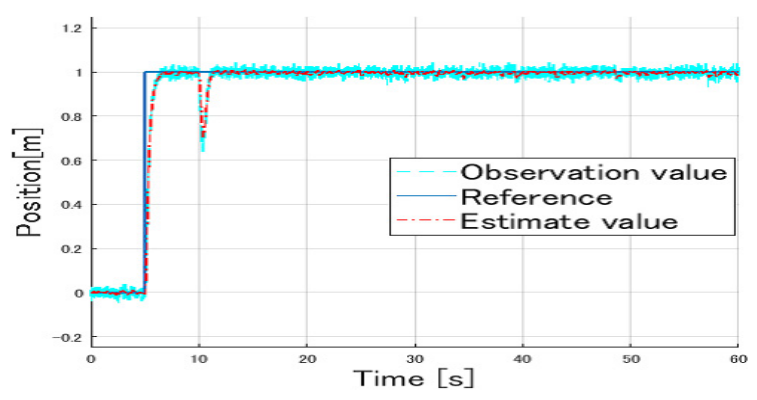

Fig. 15. Simulation results of cart position (disturbance: impulse)

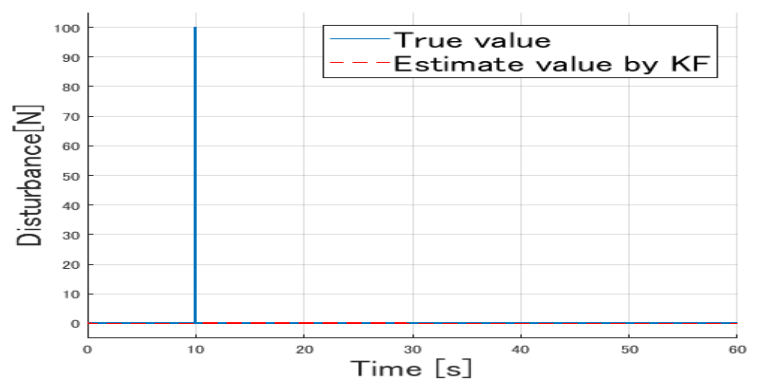

Fig. 16. Simulated disturbance estimation (disturbance: impulse)

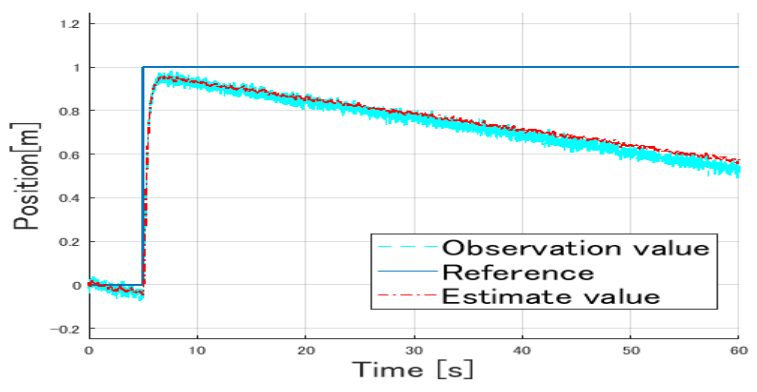

Fig. 17. Simulation results of cart position (disturbance: ramp)

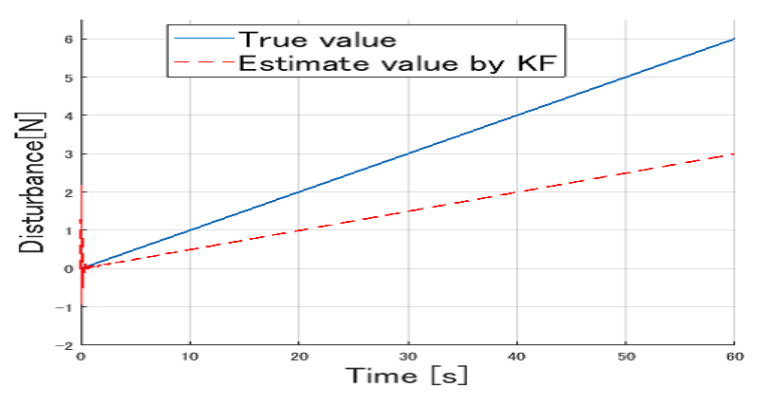

Fig. 18. Simulated disturbance estimation (disturbance: ramp)

the KF as stationary disturbances, overshoot is suppressed by the constraint on the target position, as shown in Fig. 11. Additionally, the proposed system cannot quickly remove pulse (Figs. 13 and 14) or ramp disturbances (Figs. 17 and 18). For example, in the case of ramp disturbances, it is necessary to appropriately change the expression for disturbance estimation in the enlargement system based on

$$
d(k+1)=d(k)+\dot{d}(k) .
$$

Furthermore, from Figs. 15 and 16, one see that the proposed system is only marginally affected by impulse disturbances. 


\section{Conclusion}

We proposed a control system based on MPC and a KF and verified it through simulations. In the simulations, the method was able to eliminate the influence of noise and suppressed the influence of disturbances. The proposed method also imposes constraints on the controlled system. By applying this approach, considering the influences of disturbances and noise in the control system is not required. Therefore, by controlling the system utilizing a single controller that is close to the limit of response performance for a given situation, it is possible to achieve excellent control performance and practicality. However, if an optimal solution is not found during the process of simulation, or if the modeling error or constraint infringements are large, we have confirmed that the control performance will be significantly reduced. For this reason, extending the proposed method to a robust and adaptive control system is expected to lead to an improvement in control performance.

\section{References}

(1) N. Hirose, R. Tajima, K. Sukigara, N. Oyama, M. Tanaka, and S. Ito: "Posture Stabilization Control of Personal Robot based on Model Predictive Control", IEEJ Journal of IA, Vol.135, No.3, pp.172-181 (2015) (in Japanese)

(2) T. Satoh, R. Abe, N. Saito, J, Nagase, and N. Saga: "Control of a two-link manipulator using disturbance observer-based model predictive control", Transactions of the JSME (in Japanese), Vol.81, No.827, DOI:10.1299/transjsme. 15-00084 (2015)

( 3 ) J.M. Maciejowski: "Predictive Control with Constraints", Oerson education (2002)

(4) J. Yang, S. Li, X. Chen, and Q. Li: "Disturbance rejection of ball mill grinding circuits using DOB and MPC", Powder Technology, Vol.198, No.2, pp.219-228 (2010)

( 5 ) J. Yang, W.X. Zheng, S. Li, B. Wu, and M. Cheng: "Design of a PredictionAccuracy-Enhanced Continuous-Time MPC for Disturbed Systems via a Disturbance Observer", IEEE Trans. on Industrial Electronics, Vol.62, No.9, pp.5807-5816 (2015)

(6) A. Shimada: "Application of Kalman filter type disturbance observer to an inverted pendulum type robot", The 32th Annual Conference of THE ROBOTICS SOCIETY OF JAPAN, RSJ2014AC1D1-01 (2014) (in Japanese)

( 7 ) S. Adachi and I. Maruta: "Fundamentals of Kalman Filter", Tokyo Denki University Press (2012) (in Japanese)

( 8 ) L. Wang: "Model Predictive Control System Design and Implementation Using MATLAB (Advances in Industrial Control)", Springer (2009)
( 9 ) B.M. Kapernick: "Gradient-Based Nonlinear Model Predictive Control with Constraint Transformation for Fast Dynamical Systems: 1 (Berichtea aus Steuerungs- und Regelungstechnik)", Shaker Verlag GmbH (2016)

(10) U. Maede and, M. Morari: "Offset-free reference tracking with model predictive control", Automatica, Vol.46, No.9, pp.1469-1476 (2010)

(11) K.R. Muskea and T.A. Badgwell: "Disturbance modeling for offset-free linear model predictive control", Journal of Process Control, Vol.12, No.5, pp.617-632 (2002)

(12) G. Pannocchia and J.B. Rawlings: "Disturbance models for offset-free model-predictive control", AIChE Journal, Vol.49, No.2, pp.426-437 (2003)

(13) U. Maeder, F. Borrelli, and M. Morari: "Linear offset-free Model Predictive Control", Automatica, Vol.45, No.10, pp.2214-2222 (2009)

(14) J. Nocedal and S.J. Wright: "Sequential Quadratic Programming, Numerical Optimization, Second Edition", Springer Series in Operations Research, Springer Verlag, Chapter 18, pp.529-562 (2006)

(15) M. Kitamura: "Optimization by mathematical programming", Ways of thinking and solving actual problems, Morikita Publishing Co., Ltd. (2015) (in Japanese)

(16) MathWorks Inc.: Constrained Nonlinear Optimization Algorithms, https://jp. mathworks.com/help/optim/ug/constrained-nonlinear-optimizationalgorithms.html?lang=en (accessed 2018-3-18)

(17) H. Fujimoto: "Motion Control Based on Multirate Sampling Control", IEICE Transactions on Fundamentals of Electronics, Communications and Computer Sciences, Vol.86, No.4, pp.270-276 (2003) (in Japanese)

Takashi Ohhira (Non-member) received the B.E. and M.E. degrees

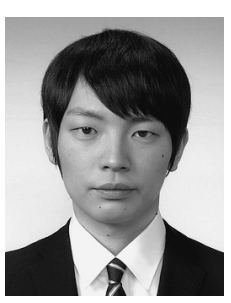
from Shibaura Institute of Technology, Japan, in 2016 and 2018, respectively. He is interested in the research about motion control for robotics. He is a member of RSJ and JSME.

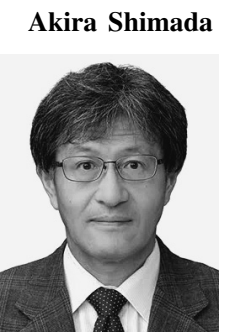

(Senior Member) received the B.S. degree in electronics engineering from the University of Electro Communications, Japan, in 1983 and received the $\mathrm{Ph} . \mathrm{D}$. in engineering from Keio University, Japan in 1996. He worked at Seiko Instruments as a robotics engineer from 1983 to 2001. Concurrently, he was a guest professor at Chiba University. He was a associate professor at the Polytechnic University, Japan from 2001 to 2009 . He has been a full professor at Shibaura Institute of Technology since 2009. He is a member of SICE, RSJ and IEEE. 